\title{
Original $\mid$ RETROSPECTIVE ANALYSIS OF PROGNOSTIC FACTORS FOR PATIENTS Article WITH BRAIN METASTASES
}

\author{
Zeinab M. Abdel Hafeez ${ }^{1}$, Hesham Elwekeel ${ }^{1}$, Reham Safwat ${ }^{2}$ and Eman T Elsheikh ${ }^{1}$
}

${ }^{1}$ Department of Clinical Oncology, Ain Shams University, ${ }^{2}$ Department of Clinical Oncology, Zakazik University, Cairo, Egypt

\begin{abstract}
Aim of the Work: To analyze the factors that affect survival in patients with brain metastases who received whole brain radiotherapy (WBRT) and to evaluate the application of the Graded Prognostic Assessment (GPA) index for these patients.

Patients and Methods: We retrospectively analyzed the data of patients who had brain metastases from solid tumors and received WBRT. The impact of different prognostic factors and GPA index on survival of these patients was estimated.

Results: Between January 2005 and December 2008, 121 patients with brain metastases were treated with WBRT. The majority of patients were females (64.5\%). About $60 \%$ of patients were $\geq 50$ years and $67 \%$ of patients had a KPS $\geq 70$. The most frequent primary tumors were breast cancer (43\%) followed by lung cancer (38.8\%) and most patients had extracranial metastases (76.9\%). The number of brain lesions exceeded 3 in $52.1 \%$ of patients. The median overall survival (OS) was 5.00 months (95\% confidence interval (CI) 4.455.55-) and the estimated 1year OS was $19.2 \%$ with (95\% CI 11.826.7-). The GPA index analysis showed strong correlation with survival ( $\mathrm{p}=0.001)$, GPA $0,1-$ 3.00 months (95\% CI 2.203.80-), GPA 1.55 .00 ,2.5- months (95\% CI 4.58- 5.42), GPA 314 ,4- months (95\% CI 11.8816.12). In univariate analysis significant prognostic factors associated with better survival were: Female sex $(p=0.028)$, KPS $\geq 70(p=0.001)$, single metastatic lesion $(p=0.034)$, brain metastases from breast cancer $(p=0.001)$. Multivariate analysis confirmed theses prognostic factors to be significantly associated with better survival.

Conclusion: The results of this study confirmed the validity of GPA index to justify its incorporation in future randomized studies on patients with brain metastases. Further examination of the applicability of this index in brain metastases from specific primary tumors is recommended.
\end{abstract}

Key Words: Metastases, prognostic factors, graded prognostic assessment.

Corresponding Author: Zeinab M. Abdel Hafeez Tel.: 0106299962 E-mail: z_hafeez@hotmail.com

\section{INTRODUCTION}

Brain metastases are the most common form of intracranial neoplasms and occur in about $25 \%$ of all cancer patients ${ }^{1}$. Historically, the prognosis was uniformly poor, with median survival time without treatment in the range of 2-4 month ${ }^{2}$. With increasing numbers of local and systemic options, the issue of patient selection gains importance and clinicians are often faced with difficult decisions regarding which treatment is appropriate for a given patient.

Prognostic indices might represent a useful tool in palliative cancer treatment. Estimation of a patient's prognosis in terms of overall survival might allow for tailored treatment in order to maximize survival and neurological function whilst avoiding unnecessary treatment. In addition, prognostic indices might be used as inclusion/exclusion criteria for clinical trials and for comparison of results across different studies in relatively homogeneous patient groups ${ }^{3,4}$. Demographic and clinical variables predictive of survival in patients with brain metastases have been well studied and important variables include: Age, performance status (PS), number of brain metastases, primary tumor type and systemic tumor activity ${ }^{5-7}$.

A new prognostic index for patients with brain metastases based on analysis of data from five randomized Radiation Therapy Oncology Group (RTOG) trials on treatment of 1960 patients with brain metastases was published by Sperduto et al. ${ }^{8}$. They aimed at defining the most useful prognostic score by comparing the already well known recursive partitioning analysis (RPA) with three classes: I (patients $<65$ years, KPS $\geq 70$, controlled primary tumor and no extracranial metastases), class III $($ KPS $<70)$ and class II (all patients not in class I or III) the Score Index for Radiosurgery, which is the sum of scores (0-2) for each of five prognostic factors (age, KPS, status of systemic disease, number of lesions and largest lesion volume $)^{9}$ and the Basic Score for Brain Metastases (BSBM), which is the sum of scores (0-1) for three prognostic factors (KPS, control of primary tumor and extracranial metastases) ${ }^{10}$. Sperduto et al. arrived at a new 
score, the Graded Prognostic Assessment (GPA) score. In the GPA index, each of 4 clinical criteria (age, Karnofsky Performance Status, number of brain metastases and presence of extracranial metastases) is given a score of $0,0.5$, or 1.0 (Table 1). A patient with the best prognosis would have a GPA score of 4 . The authors concluded that GPA is prognostically as RPA and more prognostic than the other indices and it is the least subjective, most quantitative and easiest to use of the four indices.

Therefore, we carried out this retrospective analysis to evaluate the validity of GPA in patients with brain metastases. We also analyzed the influence of different prognostic factors on survival in these patients.

\section{PATIENTS AND METHODS}

\section{Patient Characteristics:}

This retrospective study included all patients who were diagnosed with brain metastases from solid malignancies and treated with WBRT between January 2005 and December 2008 at Clinical Oncology and Nuclear Medicine Department, Ain Shams University. The patients' records were examined and those with incomplete medical data were excluded. Brain metastases were detected by contrast-enhanced brain computed tomography (CT) and/or magnetic resonance imaging (MRI). All patients had histologic confirmation of their primary malignancy or in case of unknown primary tumor a stereotactic brain biopsy was taken. The following variables were analyzed for survival: Age, sex, KPS ${ }^{11}$, number of brain metastases, presence of extracranial metastases, type of primary malignancy, interval between diagnosis of primary malignancy and development of brain metastases. Graded Prognostic Assessment (GPA) index (Table 1) was applied to all patients. All patients received WBRT using Co 60 gamma rays or 4-6 MV photons from linear accelerator. The whole brain was irradiated by opposed lateral fields which encompassed the cranium with $1 \mathrm{~cm}$ margin. The total dose ranged between 30-40 Gy in daily fractions of 3-2 Gy. Corticosteroids in individual doses were administered during radiotherapy. None of the patients was treated with radiosurgery.

\section{Statistical Analysis:}

The collected data was revised, coded, tabulated and introduced to a PC using Statistical package for Social Science (SPSS 15.0.1 for windows; SPSS Inc, Chicago, IL, 2001). Data was presented and suitable analysis was done according to the type of data obtained for each parameter. Overall survival (OS) was the end point of analysis for prognostic factors and GPA index. Overall survival was calculated from the date of WBRT start to the date of death or last follow up using the KaplanMeier method ${ }^{12}$. Survival curves were compared using the log-rank test. All factors with a p-value of $\leq 0.05$ at univariate analysis were entered into multivariate cox regression analysis. Hazard ratios and 95\% confidence intervals were calculated. Significance level of $\leq 0.05$ was used throughout all statistical tests in this study.

Table 1: Graded Prognostic Assessment,

\begin{tabular}{lccc}
\hline & \multicolumn{3}{c}{ Score } \\
\hline & 0 & 0.5 & 1 \\
Age & $>60$ & $50-59$ & $<50$ \\
KPS & $<70$ & $70-80$ & $90-100$ \\
$\begin{array}{l}\text { No. of CNS metastases } \\
\begin{array}{l}\text { Extracranial } \\
\text { metastases }\end{array}\end{array}$ & $>3$ & $2-3$ & 1 \\
\hline
\end{tabular}

KPS $=$ Karnofsky Performance Status, $\mathrm{CNS}=$ Central Nervous System

\section{RESULTS}

\section{Patient Characteristics:}

Between January 2005 and December 2008, 121 patients with brain metastases from solid tumors were treated with WBRT at our department. The majority of patients were females ( 78 patients). About $60 \%$ of patients were $\geq 50$ years and most patients (81) had a KPS $\geq 70$. The most frequent primary tumors were breast cancer (52 patients) and lung cancer (47 patients). Most patients had extracranial metastases (93 patients). All patients underwent CT brain before WBRT while MRI was done for 89 patients. In 12 patients stereotactic or surgical biopsy was taken from the brain lesions to establish the diagnosis. Number of brain lesions exceeded 3 in 63 patientshh. All patients received at least $30 \mathrm{~Gy}$ WBRT dose. Patients were classified according to GPA scoring index. As 3 patients only had GPA score 3 and for purpose of statistical analysis patients with scores 3-4 were grouped together. The characteristics of patients are shown in (Table 2).

$\mathrm{KPS}=\quad$ Karnofsky Performance Status, $\mathrm{NSCLC}=$ Non Small Cell Lung Cancer, SCLC $=$ Small Cell Lung Cancer, GPA= Graded Prognostic Assessment

\section{Survival Analysis:}

Median survival time for the whole group of patients $(n=121)$ was 5.00 months $(95 \%$ CI 4.45-5.55) and the estimated one year survival was $19.2 \%$ with $(95 \%$ CI 11.8-26.7) (Figure 1). The GPA index analysis showed strong correlation with survival $(\mathrm{p}=0.001)$, GPA 0-1, 3.00 months (95\% CI 2.20-3.80), GPA 1.5-2.5, 5.00 months (95\% CI 4.58- 5.42), GPA 3-4, 14 months (95\% CI 11.88-16.12) (Figure 2). In univariate analysis significant prognostic factors associated with better survival were: Female sex $(p=0.028), K P S \geq 70 \quad(p=0.001)$, single metastatic lesion $(p=0.034)$ and brain metastases from breast cancer $(p=0.001)$. Multivariate analysis confirmed theses prognostic factors to be significantly associated with better survival. 
Table 2: Characteristics of study patients $(n=121)$.

\begin{tabular}{|c|c|c|}
\hline Variable & No & $\%$ \\
\hline \multicolumn{3}{|l|}{ Age } \\
\hline$\geq 60$ & 24 & 19.8 \\
\hline $50-59$ & 50 & 41.3 \\
\hline$<50$ & 47 & 38.8 \\
\hline \multicolumn{3}{|l|}{ Sex } \\
\hline Male & 43 & 35.5 \\
\hline Female & 78 & 64.5 \\
\hline \multicolumn{3}{|l|}{ KPS } \\
\hline$<70$ & 40 & 33.1 \\
\hline $70-80$ & 62 & 51.2 \\
\hline $90-100$ & 19 & 15.7 \\
\hline \multicolumn{3}{|l|}{ Primary tumor site } \\
\hline Breast & 52 & 43.0 \\
\hline NSCLC & 28 & 23.1 \\
\hline SCLC & 19 & 15.7 \\
\hline Other & 21 & 17.3 \\
\hline \multicolumn{3}{|c|}{ Extracanial metastases } \\
\hline Yes & 93 & 76.9 \\
\hline No & 28 & 23.1 \\
\hline \multicolumn{3}{|c|}{$\begin{array}{l}\text { Interval from initial diagnosis till brain } \\
\text { metastases in months }\end{array}$} \\
\hline$\leq 12$ & 62 & 51.2 \\
\hline $13-36$ & 32 & 24.8 \\
\hline$>36$ & 27 & 7.4 \\
\hline \multicolumn{3}{|l|}{ Primary tumor } \\
\hline Controlled & 28 & 23.1 \\
\hline Uncontrolled & 93 & 76.9 \\
\hline \multicolumn{3}{|c|}{ Number of brain lesions } \\
\hline$>3$ & 63 & 52.1 \\
\hline $2-3$ & 22 & 18.2 \\
\hline 1 & 36 & 29.8 \\
\hline \multicolumn{3}{|l|}{ GPA } \\
\hline $0-1$ & 63 & 52.1 \\
\hline $1.5-2.5$ & 32 & 26.4 \\
\hline $3-4$ & 26 & 21.5 \\
\hline
\end{tabular}

KPS $=$ Karnofsky Performance Status, NSCLC $=$ Non Small Cell Lung Cancer, $\mathrm{SCLC}=$ Small Cell Lung Cancer, GPA $=$ Graded Prognostic Assessment

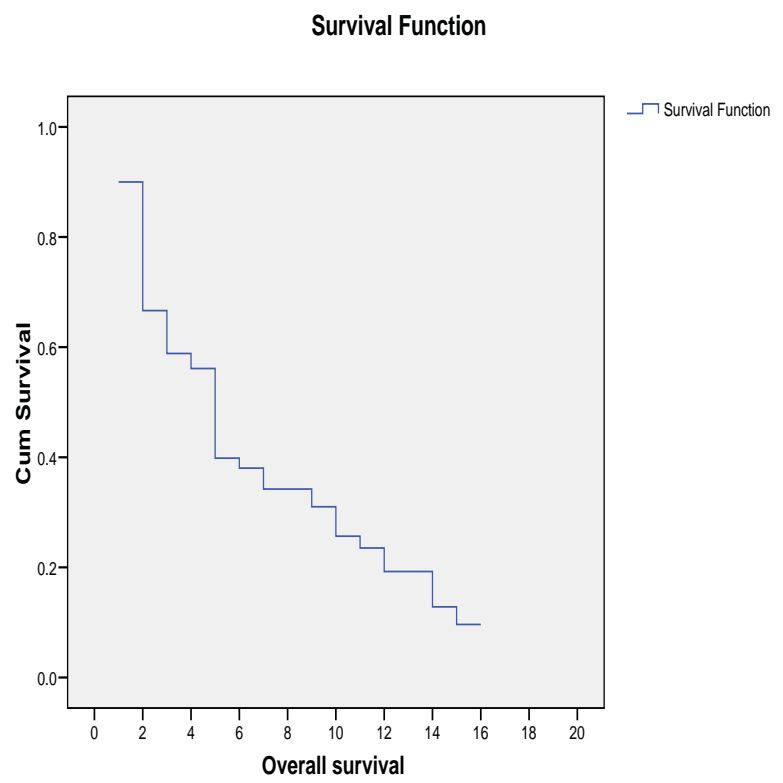

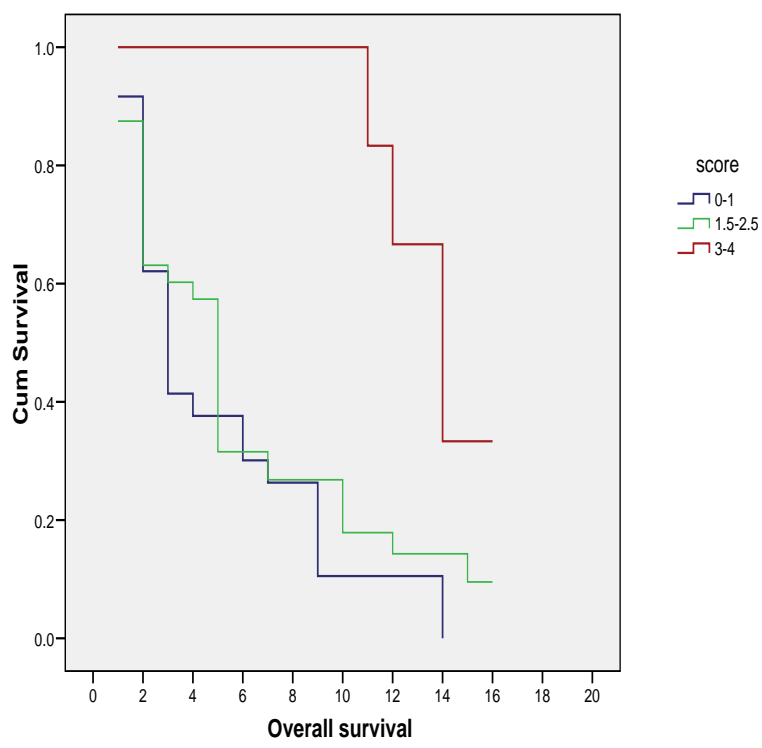

Figure 2: Kaplan-Meier curves according to GPA index.

\section{DISCUSSION}

Brain metastases are a common and challenging problem in clinical oncology today. In recent years, with improvement in imaging and evolution of treatments such as SRS, it has become clear that not all patients with brain metastases have the same prognosis and the use of the same treatment for all patients is no longer appropriate ${ }^{8}$. Treatment of brain metastases depends on their number, location, age of the patient, performance status, presence of extra-cerebral lesions and a prediction of their response to systemic therapy. An adequate estimation of these prognostic factors is required to enable the clinician to decide between invasive treatments and to avoid unnecessary treatment ${ }^{1}$.One of the purposes of prognostic indices is to guide the choice of treatment in individual patients. In this context a prognostic index should be accurate enough to avoid overtreatment in patients that actually have very short survival. Another point is to identify patients before treatment who would benefit or not from such therapy, not at the time the therapy is delivered ${ }^{3}$.

A new prognostic index, GPA, was proposed by RTOG in the light of new data being available from the RTOG 9508 trial and also to overcome the limitations of previously proposed indices. The limitations of the previous indices include:

1. The RPA and BSBM do not incorporate number of metastases;

2. All three previous indices require the estimation of control of systemic disease, which is fraught with inconsistency because of variation in type and timing of imaging tests.

3. The SIR requires treatment factors (volume of the largest lesion at the time of radiosurgery) when the point of prognostic indices is to predict outcome before, not after.

Figure 1: Kaplan-Meier overall survival curve. 
Treatment decisions are made, thus guiding the decisions. This index was found to be less subjective and easier to use ${ }^{8}$.

In the current study we tested the validity of GPA retrospectively on 121 patients with brain metastases from solid tumors who were treated with WBRT. As 3 patients only had GPA score 3 and for purpose of statistical analysis patients with scores 3-4 were grouped together. In spite of the small number of patients in this study, the GPA index analysis showed strong correlation with median survival ( $\mathrm{p}=0.001$ ), GPA $0-1,3.00$ months, GPA 1.5-2.5, 5.00 months and GPA 3-4, 14 months. In the original study of Sperduto et al. ${ }^{8}$, median survival times for the GPA 0-1, 2.6 months, GPA1.5-2.5., 3.8 months, GPA 3, 6.9 months and GPA 3.5-4.0, 11.0 $(\mathrm{p}<0.0001)^{8}$. Nieder et al. ${ }^{3}$, confirmed the validity of the GPA index in 232 patients with brain metastases and they reported median values for GPA groups of 1.9 months, 3.5 months, 5.6 months and 10.3 months, respectively ${ }^{13}$. The relatively better survival of patients in the present study may be explained by the small number of patients and the selection bias, which is almost always a limitation in all retrospective studies.

Analysis of different prognostic factors in our patients revealed that significant prognostic factors associated with better survival, other than GPA index score were: Female $\operatorname{sex}(p=0.028), K P S \geq 70(p=0.001)$, single metastatic lesion $(\mathrm{p}=0.034)$ and brain metastases from breast cancer $(p=0.001)$. Multivariate analysis confirmed these prognostic factors to be independently associated with better survival. Many studies confirmed the importance of KPS, number of brain metastases and primary tumor type as prognostic factors in patients with brain metastases ${ }^{5-8,14}$. Of these, the KPS score has consistently been shown to be the major determinant of survival, secondary only to treatment regimen in most studies ${ }^{5-8}$. The improved survival for female patients in the current study is most probably a reflection of better survival for brain metastases from breast cancer in our patients; all of them received systemic therapy. However, complete analysis of clinicopathologic and biologic data of these patients was not possible in this study. In a recently published study conducted by Niwinska et al. ${ }^{15}$, on 222 patients with breast cancer metastatic to the brain, they found that survival depended on biologic subtype, performance status, RPA class and the use of systemic treatment ${ }^{15}$.

In conclusion and in spite of the limitation of this study, being retrospective and on a small number of patients, it confirms the validity of GPA index and its ease in application. It is recommended to incorporate this index in prospective clinical trials on patients with brain metastases. However, in making treatment decisions, caution should be used when applying any general prognostic grading system for brain metastases to individual patients as the applicability of prognostic grading systems may vary by primary tumor site.

\section{REFERENCES}

1. Wadasadawala T, Gupta S, Bagul V, Patil N. Brain metastases from breast cancer: Management approach. J.Cancer Res. Therap. 2007;3(3):157-65.

2. Weissman DE. Glucocorticoid treatment for brain metastases and epidural spinal cord compression: A review. J.Clin. Oncol. 1988 Mar;6(3):543-51.

3. Nieder C, Mehta MP. Prognostic indices for brain metastases - Usefulness and challenges. Radiat.Oncol. 2009;4:Art. No. 10.

4. Eichler AF, Loeffler JS. Multidisciplinary management of brain metastases. Oncologist 2007;12(7):884-98.

5. Gaspar L, Scott C, Rotman M, Asbell S, Phillips T, Wasserman T, et al. Recursive Partitioning Analysis (RPA) of prognostic factors in three Radiation Therapy Oncology Group (RTOG) brain metastases trials. Int.J.Radiat.Oncol. Biol.Phys. 1997 Mar 1;37(4):745-51.

6. Lagerwaard FJ, Levendag PC, Nowak PJ, Eijkenboom WM, Hanssens PE, Schmitz PI. Identification of prognostic factors in patients with brain metastases: A review of 1292 patients. Int.J.Radiat.Oncol.Biol.Phys. 1999 Mar 1;43(4):795-803.

7. Fife KM, Colman MH, Stevens GN, Firth IC, Moon D, Shannon KF, et al. Determinants of outcome in melanoma patients with cerebral metastases. J.Clin.Oncol. 2004 Apr 1;22(7):1293-300.

8. Sperduto PW, Berkey B, Gaspar LE, Mehta M, Curran W. A new prognostic index and comparison to three other indices for patients with brain metastases: An analysis of 1,960 patients in the RTOG database. Int.J.Radiat.Oncol.Biol. Phys. 2008;70(2):510-4.

9. Weltman E, Salvajoli JV, Brandt RA, de Morais Hanriot R, Prisco FE, Cruz JC, et al. Radiosurgery for brain metastases: A score index for predicting prognosis. Int.J.Radiat.Oncol. Biol.Phys. 2000 Mar 15;46(5):1155-61.

10. Lorenzoni J, Devriendt D, Massager N, David P, Ruíz $\mathrm{S}$, Vanderlinden $\mathrm{B}$, et al. Radiosurgery for treatment of brain metastases: Estimation of patient eligibility using three stratification systems. Int.J.Radiat.Oncol.Biol.Phys. 2004;60(1):218-24.

11. Yates JW, Chalmer B, McKegney FP. Evaluation of patients with advanced cancer using the Karnofsky performance status. Cancer 1980 Apr 15;45(8):2220-4.

12. Kaplan EL, Meier P. Non parametric estimation from incomplete observations. J.Am.Stat.Assoc. 1958;53(282):457-81.

13. Nieder C, Marienhagen K, Geinitz H, Molls M. Validation of the graded prognostic assessment index for patients with brain metastases. Acta Oncol. 2009;48(3):457-9.

14. Golden DW, Lamborn KR, McDermott MW, Kunwar S, Wara WM, Nakamura JL, et al. Prognostic factors and grading systems for overall survival in patients treated with radiosurgery for brain metastases: Variation by primary site. J.Neurosurg. 2008;109 Suppl.:77-86.

15. Niwiñska A, Murawska M, Pogoda K. Breast cancer brain metastases: Differences in survival depending on biological subtype, RPA RTOG prognostic class and systemic treatment after whole-brain radiotherapy (WBRT). Ann. Oncol. 2009;21(5):942-8. 